\title{
ReMed: Jahresbericht 2014
}

ReMed, Leitungsausschuss

\section{ReMed Schweiz - Peter Christen neuer Programmleiter}

Das Unterstützungsnetzwerk hat einen neuen Programmleiter. Anfang Juli hat Dr. med. Michael Peltenburg, der Mitbegründer von ReMed, seine Leitungsaufgaben an Dr. med. Peter Christen übergeben. Herr Dr. Christen verfügt über eine breite Praxiserfahrung in Hausarztmedizin mit Weiterbildung in Psychotherapie und Psychosomatik und erfüllt somit die Voraussetzungen für die Programmleitung in optimaler Weise. Ebenso wurde per Juli mit Dr. med. Franco Gusberti ein weiteres Mitglied in den Leitungsausschuss gewählt. Den Verantwortlichen von ReMed ist es ein Anliegen, dass die jungen Ärztinnen und Ärzte, die in letzter Zeit immer mehr die Unterstützungsangebote von ReMed in Anspruch nehmen, auch adäquat im Leitungsteam vertreten sind. Man wird deshalb bestrebt sein, 2015 den Leitungsausschuss um weitere Personen aus dem Kreis der jungen Ärzteschaft zu erweitern bzw. zu ergänzen. Der Leitungausschuss setzt sich wie folgt zusammen:

\section{Programmleitung}

- Dr. med. Peter Christen, Facharzt für Allgemeine Innere Medizin, FMH, ärztliche Programmleitung;

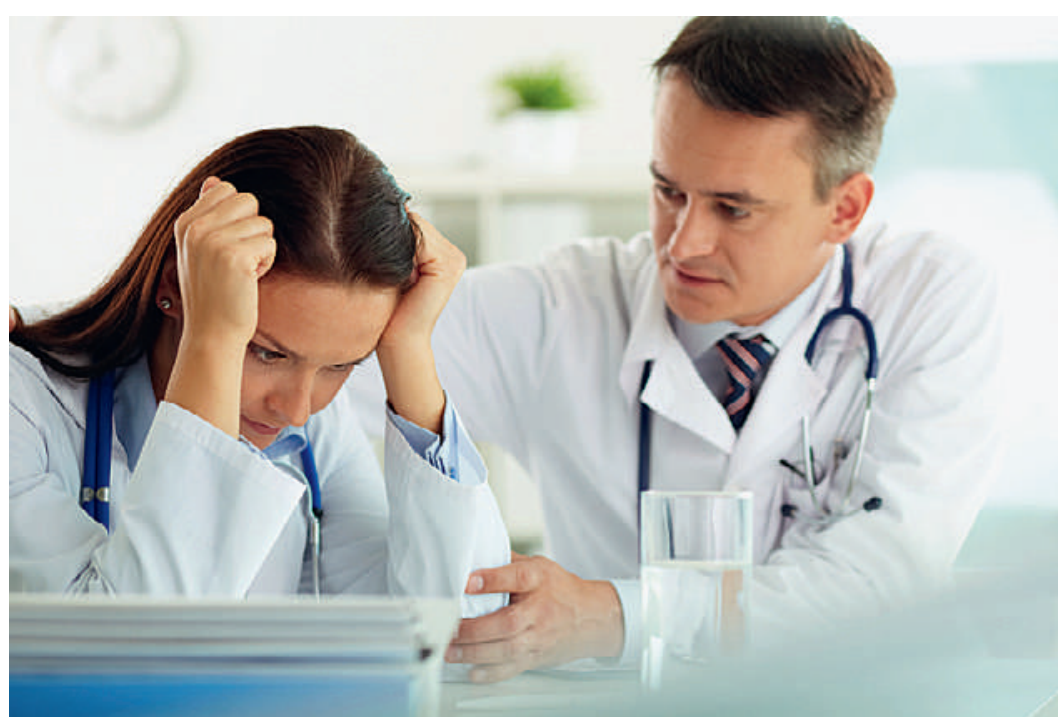

Vor allem Ärztinnen und Ärzte in Spitälern wurden 2014 auf das Unterstützungsangebot von ReMed aufmerksam gemacht.
- Esther Kraft, lic. rer. oec., Leiterin Abteilung Daten, Demographie und Qualität FMH, administrative Programmleitung.

\section{Leitungsausschuss}

- Dr. med. Jürg. Bänninger, Facharzt für Allgemeine Innere Medizin, FMH;

- Dr. med. Peter Birchler, Facharzt für Psychiatrie und Psychotherapie, FMH;

- Dr. med. Franco Gusberti, Facharzt für Psychiatrie und Psychotherapie, FMH;

- Dr. med. Susanne Meier, Fachärztin für Allgemeine Innere Medizin, FMH;

- Dr. med. Mirjam Tanner, Fachärztin für Psychiatrie und Psychotherapie, FMH;

- Dr. med. Michael Peltenburg, Facharzt für Allgemeine Innere Medizin, FMH

\section{ReMed-Programm}

An Kongressen im Inland (u.a. Burnout-Symposium Kilchberg und Public Health Kongress in Olten) als auch im Ausland [International Conference on Physician Health (ICPH) in London] machte der Leitungsausschuss wiederum auf das Unterstützungsnetzwerk ReMed und dessen Angebot aufmerksam. Es wurden wichtige Inputs gegeben und zahlreiche interessante Kontakte $\mathrm{zu}$ anderen Organisationen geknüpft, welche weiterverfolgt werden. Auch im laufenden Jahr wird ReMed wieder an Veranstaltungen teilnehmen und das Programm und dessen Angebot präsentieren. ReMed wurde 2014 über 80-mal kontaktiert - interessanterweise erfolgte ca. ein Viertel der Kontaktaufnahmen am Donnerstag.

\section{Netzwerk festigen}

\section{Intervisionen Deutschschweiz}

Die Intervisionstreffen in der Deutschschweiz, die seit Einführung des Programms bestehen, sind auch im vergangenen Jahr erfolgreich umgesetzt worden. Das Netzwerk von den Kollegen und Kolleginnen, das Coaching, Beratung, Therapie und professionelle Unterstützung anbietet, hat sich 2014 weiter gefestigt. Die Themen der Treffen werden nicht durch ReMed vorgegeben. Das erklärte Ziel der Intervision 
in der Deutschschweiz ist es, von Anfang an mit den Fragen, Anliegen und Themen zu arbeiten, fallbezogen oder auch nicht, die jeweils durch die Teilnehmer eingebracht werden. Der Leitungsausschuss ist bestrebt, das Netz weiter zu vergrössern und weitere Ärztinnen und Ärzte gewinnen zu können. Interessenten sind willkommen.

\section{Rencontre Romandie}

Die Netzwerkpartner der Romandie und Vertreter des Leitungsausschusses haben sich im letzten Jahr dreimal getroffen. Die Treffen waren Voraussetzung für die Einführung der interaktiven Theater in der

\section{Betroffene Ärztinnen und Ärzte können sich über die 24-Stunden-Telefon-Hotline oder per E-Mail melden.}

Romandie sowie deren gutes Gelingen. Die Treffen dienten auch als Plattform für den Austausch zwischen den Ärztinnen und Ärzten in der Romandie, welche in der Beratung tätig waren.

\section{Deutlich machen}

Mit einem Grossversand von Flyern und Kleinplakaten an alle Qualitätsverantwortlichen wurden die Spitäler Anfang 2014 wiederum auf das Angebot von ReMed aufmerksam, das somit den Ärztinnen und Ärzten näher gebracht wurde. Auch die in der Schweizerischen Ärztezeitung veröffentlichten Erfahrungsberichte zeigten auf, wie ReMed Betroffenen in Notfallsituationen Hilfe anbietet und Lösungen findet. Grosse Reaktionen fand der Erfahrungsbericht «Sexuelle Übergriffe: ReMed verurteilt nicht" (SÄZ 42/2014). Mittels einer Replik (SÄZ 1-2/2O15) ist ReMed auf die zahlreichen Rückmeldungen eingegangen und stellte die Haltung und Handlungsmöglichkeiten bei Übergriffen, insbesondere sexuellen, differenziert und ausführlich dar. Das oben erwähnte Testimonial hat zur weiteren Sensibilisierung dieses immer noch tabuisierten Themas beigetragen und ReMed als niederschwellige und als präventive Anlaufstelle weiter bekannt gemacht.

\section{Sichtbar werden}

Wie in den Vorjahren fanden auch 2014 Aufführungen mit dem interaktiven Theater Knotenpunkt statt. Die Veranstaltungen in Basel und am Public Health Kongress in Olten waren zahlreich besucht und fanden beim Publikum einen grossen Anklang. Ebenso erfreulich ist es, dass mit der Theatergruppe «Le Caméléon» ein Pendant für die Romandie erarbeitet wurde. Die erste Aufführung vom Dezember 2014 in Neuenburg fand grossen Anklang beim Publikum. Durch gezielte Moderation werden bei beiden Darbietungen die Zuschauenden animiert, die Handlungsweisen der Figuren auf der Bühne nach den eigenen Vorstellungen zu gestalten. Das Publikum greift aktiv ins Bühnengeschehen ein und probiert seine Sicht der Dinge direkt aus. So erleben die Teilnehmer unmittelbar die Folgen ihres eigenen Handelns.

\section{Weiterentwickeln}

Das nationale Programm ReMed mit dem Leitungsausschuss und den Netzwerkpartnern wird auch im Jahr 2015 für Ärztinnen und Ärzte in Krisensituationen da sein. Die betroffenen Ärztinnen und Ärzte können sich über die 24-Stunden-Hotline (o80o o 73633) oder per help[at]swiss-remed.ch melden. Ein erfahrenes Beraterteam von Ärztinnen und Ärzten bespricht mit der betroffenen Person die persönliche Situation innert 72 Stunden

Selbstverständlich bietet ReMed auch 2015 wiederum die Intervisionstreffen an, lanciert/organisiert in der Romandie und Deutschschweiz die interaktiven Theater, präsentiert sich an Anlässen/Kongressen und macht mit den Erfahrungsberichten auf das Angebot von ReMed aufmerksam. 